Paper

\title{
A novel hardware-efficient gene network model based on asynchronous cellular automaton dynamics
}

\author{
Takuya Yoshimoto $^{1}$ and Hiroyuki Torikai ${ }^{1 a)}$ \\ ${ }^{1}$ Department of Computer Science, Kyoto Sangyo University \\ Kyoto 603-8555, Japan \\ a)torikai@cse.kyoto-su.ac.jp
}

Received January 24, 2017; Revised May 27, 2017; Published October 1, 2017

\begin{abstract}
The gene affects various behaviors of animals such as circadian rhythm, courtship behavior, motor behavior, visual behavior, and learning. The circadian rhythm is a biological rhythm having a period of almost 24 hours, which is sometimes called internal clock or biological clock. In this paper, a novel asynchronous cellular automaton model of a gene network is proposed, where its vector field is designed based on an ordinary differential equation gene network model. It is shown that the proposed model can reproduce typical phenomena (e.g., oscillations, mutual synchronization, locking to light stimulation, and related bifurcations) observed in the differential equation gene network model. It is also shown that the proposed model can be implemented on an FPGA by using much less hardware resource compared to the differential equation model
\end{abstract}

Key Words: gene network, circadian rhythm, asynchronous cellular automaton, FPGA

\section{Introduction}

The gene affects various behaviors of animals such as circadian rhythm, courtship behavior, motor behavior, visual behavior, and learning. There exist a variety of gene network models [1-7]. Among them, in this paper, a gene network model of circadian rhythm [7] is focused on since the circadian rhythm is one of the most fundamental gene-oriented phenomena. It should be emphasized that the circadian rhythm is only one example of gene-oriented phenomena and thus investigation of the circadian rhythm will be an entrance into investigations of complicated gene networks and their applications such as genomic drug discovery and genomic medicine. The circadian rhythm is a geneoriented rhythmic phenomena, which is generated by a network of suprachiasmatic nucleus (SCN) neurons [5-7]. In the single SCN neuron, a gene network forms a closed-loop nonlinear dynamical system, which leads to periodic oscillations of an mRNA concentration, a protein concentration, and a neuropeptide concentration. Reference [7] considers the case where SCN neurons have slightly different parameter values and oscillation periods of mRNA concentrations of isolated SCN neurons are slightly different. In the suprachiasmatic nucleus, the SCN neurons are coupled via the neuropeptide and the oscillation periods of the mRNA concentrations are almost identical and have a sharp distribution near 


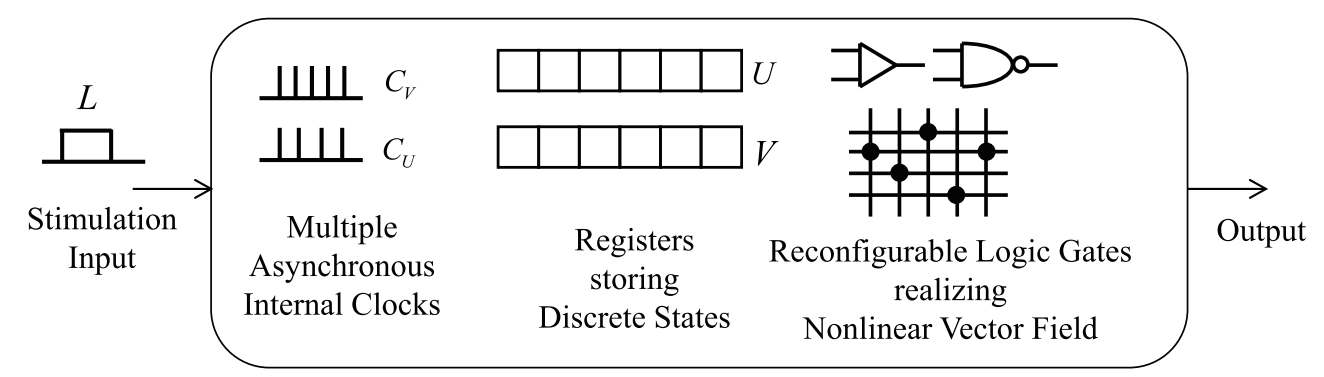

Fig. 1. Typical structure of a biological system model based on an asynchronous cellular automaton, which has a continuous (state transition) time and discrete states.

(but not identical with) 24 hours. Due to this synchronization phenomenon, the total concentration of the neuropeptide (which affects behavior of an animal) can have a much larger amplitude compared to the isolated case. So, the SCN neurons may utilize the synchronization phenomenon to strengthen the influence on the behavior. In addition, if a light-and-dark stimulation with period 24 hours is applied to a visual system, the suprachiasmatic nucleus is affected by the stimulation and then the periods of the mRNA concentrations in the coupled SCN neurons are sharply locked to 24 hours. Due to this locking phenomenon, the neuropeptide concentration can have a strong oscillation with period 24 hours. So, the SCN neurons may utilize the locking phenomenon to adjust the period of the circadian rhythm to just 24 hours.

Now, let us consider classes of biological system models. Deterministic biological system models can be classified into the following four classes based on continuousness and discontinuousness of state variables and times, where partial differential equation models are omitted for simplicity.

- Class CTCS (continuous time and continuous state):

A nonlinear differential equation model of a biological system, which has a continuous time and continuous states. Such a model can be implemented by an analog nonlinear electronic circuit.

- Class DTCS (discrete time and continuous state):

A nonlinear difference equation model of a biological system, which has a discrete time and continuous states. Such a model can be implemented by a switched capacitor circuit or by a Poincare map.

- Class DTDS (discrete time and discrete state):

A numerical integration model of a biological system, which has a discrete time and discrete states including fixed point numbers. Such a model can be implemented by a digital processor. A cellular automaton model of a biological system also belongs to this class, which has a discrete time and discrete states. Such a model can be implemented by a traditional synchronous sequential logic circuit.

- Class CTDS (continuous time and discrete state):

An asynchronous cellular automaton model of a biological system, which has a continuous (state transition) time and discrete states. Such a model can be implemented by an asynchronous sequential logic circuit (see also Fig. 1).

It goes without saying that most conventional deterministic biological system models are belonging to the classes CTCS, DTCS, and DTDS. On the other hand, our group has been developing a biological system modeling approach based on the nonlinear dynamics of an asynchronous cellular automaton, which belongs to the class CTDS system [8-15]. As illustrated in Fig. 1, such a model typically consists of uncoupled asynchronous internal clocks, registers (storing discrete state variables), logic gates (realizing a nonlinear vector field), and reconfigurable wires (realizing adjustable system parameters). Our group has shown that biological system models based on the asynchronous cellular automaton consume much less hardware resource compared to conventional ones [8-15].

So, the purpose of this paper is to propose a novel design method of a gene network model based on the nonlinear dynamics of the asynchronous cellular automaton. First, a novel asynchronous 
cellular automaton model of a gene network is proposed, where its vector field is designed based on the ordinary differential equation gene network model [7]. It is then shown that the proposed gene network model can reproduce typical phenomena (e.g., oscillations, mutual synchronization, and locking to light stimulation) observed in the ordinary differential equation gene network model [7] as well as some of their underlying bifurcation mechanisms. Note that this paper shows that a class CTDS system having more than three discrete state variables can exhibit such bifurcations for the first time. Next, the proposed gene network model is implemented on an FPGA and experiments validate its operation. It is then shown that the proposed gene network model consumes much less hardware resource compared to the ordinary differential equation gene network model. So, the results of this paper may contribute to develop other gene network models that consume less hardware resource compared to ordinary differential equation gene network models. Such a hardware-efficient gene network model will contribute to develop a hardware-based gene network analyzer, applications of which would include genetic pharmacy and genetic medicine. Note that the vector field of the proposed model is designed based on the ordinary differential equation gene network model in [7] from the following reasons. Recall that this paper presents the asynchronous cellular automaton gene network model for the first time. In such a starting point of a research, a low dimensional model like [7] would be a good candidate to be used as a reference to design the vector field. In addition, the model [7] would be a good candidate for comparison since its bifurcations are well-studied. Very preliminary and limited results of this paper can be found in conference papers [16, 17].

\section{ODE gene network model}

There are a lot of gene network models [1-7]. As a relatively simple model, in this section, an ordinary differential equation (ODE) gene network model of circadian rhythm [7] is focused on. Let

$$
t \in \boldsymbol{R}
$$

represent a continuous time and let

$$
x_{i} \in \boldsymbol{R}^{+}=\{x \mid x \in \boldsymbol{R}, x \geq 0\}, y_{i} \in \boldsymbol{R}^{+}, \quad z_{i} \in \boldsymbol{R}^{+}, \quad v_{i} \in \boldsymbol{R}^{+}
$$

represent an mRNA concentration of a clock gene in a cell, the resulting protein concentration, an inhibitor (active protein) concentration, and a neuropeptide concentration, respectively, where $i$ represents the cell index. Then the dynamics of the ODE gene network model is described by the following set of equations.

$$
\begin{aligned}
& \frac{d x_{i}}{d t}=\alpha_{1, i} \frac{\beta_{1, i}^{n}}{\beta_{1, i}^{n}+z_{i}^{n}}-\alpha_{2, i} \frac{x_{i}}{\beta_{2, i}+x_{i}}+\alpha_{c, i} \frac{k f}{\beta_{c, i}+k f}+l(t), \\
& \frac{d y_{i}}{d t}=\beta_{3, i} x_{i}-\alpha_{3, i} \frac{y_{i}}{\beta_{4, i}+y_{i}}, \\
& \frac{d z_{i}}{d t}=\beta_{5, i} y_{i}-\alpha_{4, i} \frac{z_{i}}{\beta_{6, i}+z_{i}}, \\
& \frac{d v_{i}}{d t}=\beta_{7, i} x_{i}-\alpha_{5, i} \frac{v_{i}}{\beta_{8, i}+v_{i}}, \\
& f=\frac{1}{J} \sum_{i=1}^{J} v_{i},
\end{aligned}
$$

where $J$ represents the number of cells; $f$ represents a mean field of the neuropeptide $v_{i}$;

$$
\boldsymbol{p}_{i}=\left(\alpha_{1, i}, \alpha_{2, i}, \alpha_{3, i}, \alpha_{4, i}, \alpha_{5, i}, \alpha_{c, i}, \beta_{1, i}, \beta_{2, i}, \beta_{3, i}, \beta_{4, i}, \beta_{5, i}, \beta_{6, i}, \beta_{7, i}, \beta_{8, i}, \beta_{c, i}\right)
$$

are parameters of the $i$-th cell; and $k$ is a coupling parameter. Also, $l(t)$ represents the following light stimulation with period 24 hours. 


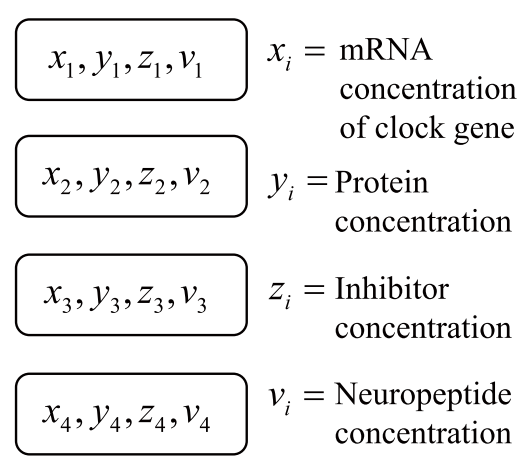

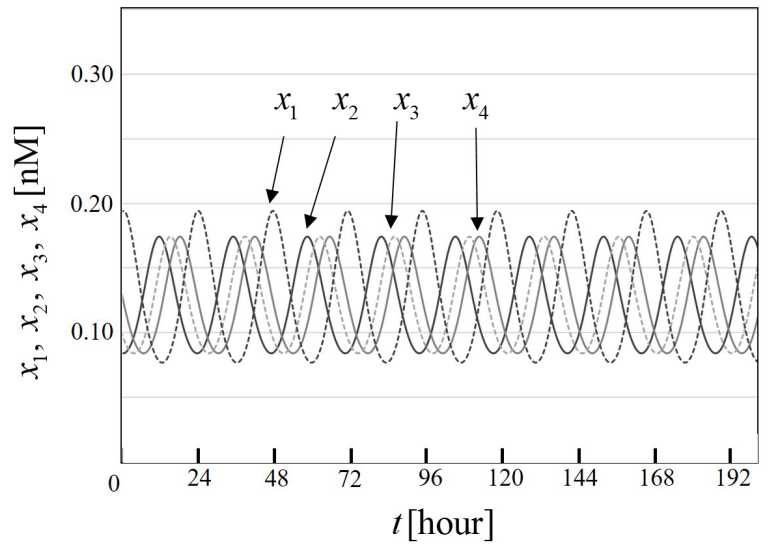

(a) Uncoupled four cells.
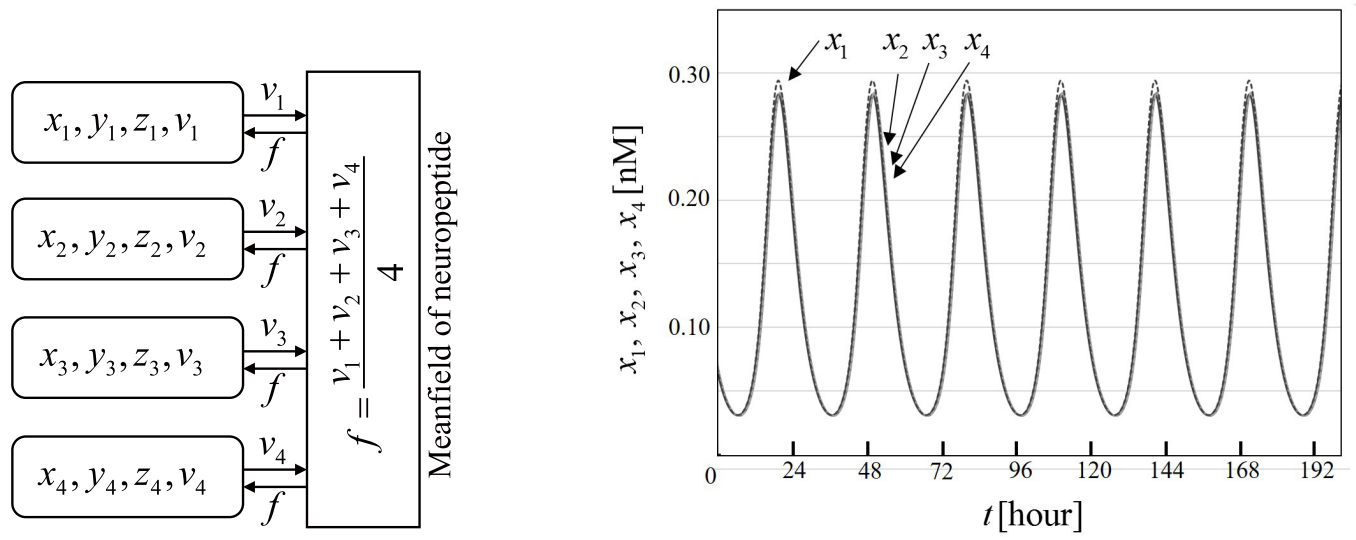

(b) Four cells coupled via mean field $f$ of neuropeptide $v_{i}$.
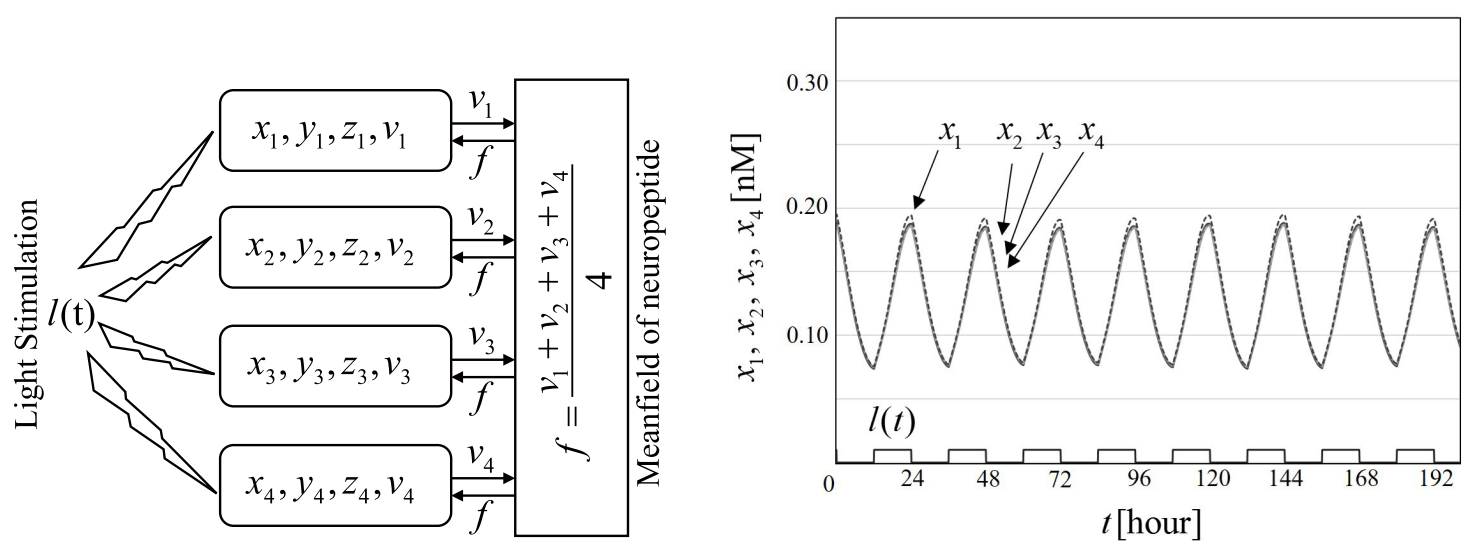

(c) Coupled four cells under light stimulation $l(t)$ with period 24 hours.

Fig. 2. Typical phenomena of the ODE gene network model [7] in Eq. (1) with $J=4$ cells. The nano molar "nM" is the unit of the molar concentration, where $1[\mathrm{nM}]$ is equivalent to $10^{-6}\left[\mathrm{~mol} / \mathrm{m}^{3}\right]$. The four cells have slightly different parameter values: $\boldsymbol{p}_{1}=(0.707,0.3535,0.3535,0.3535,1.01,0.4,1,1$, $0.707,1,0.707,1,0.3535,1,1), \boldsymbol{p}_{2}=(0.7,0.35,0.35,0.35,1,0.4,1,1,0.7$, $1,0.7,1,0.35,1,1), \boldsymbol{p}_{3}=(0.7007,0.35035,0.35035,0.35035,1.001,0.4,1,1$, $0.7007,1,0.7007,1,0.35035,1,1)$, and $\boldsymbol{p}_{4}=(0.693,0.3535,0.3535,0.3535$, $0.99,0.4,1,1,0.693,1,0.693,1,0.3535,1,1)$. (a) Oscillations. The cells are uncoupled. $k=0, l_{0}=0$. (b) Mutual synchronization. The cells are coupled via the mean field $f$ of the neuropeptide $v_{i} . k=0.5, l_{0}=0$. (c) Locking of period to stimulation period. The cells are coupled via the mean field $f$ of the neuropeptide $v_{i}$ and an external light stimulation $l$ with period 24 hours is applied to the cells. $k=0.5, l_{0}=0.01$. 


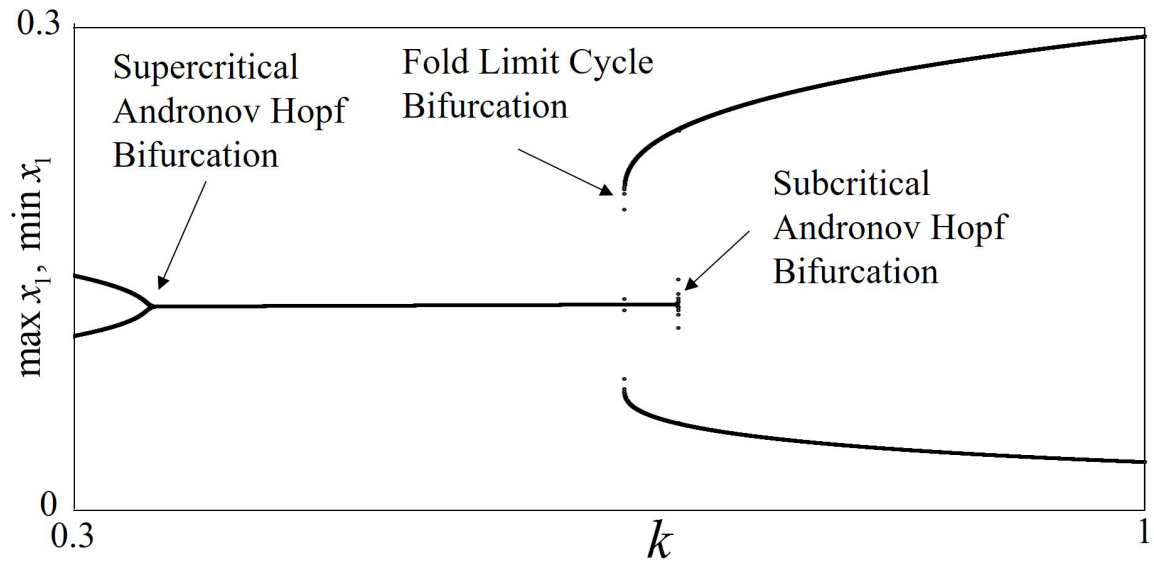

Fig. 3. One parameter bifurcation diagram of the ODE gene network model in [7] with respect to the coupling parameter $k . J=2 . \boldsymbol{p}_{1}=(0.77,0.385$, $0.385,0.385,1,0.4,1,1,0.77,1,0.77,1,0.385,1,1) . p_{2}=(0.707,0.3535$, $0.3535,0.3535,1.01,0.4,1,1,0.707,1,0.707,1,0.3535,1,1)$.

$$
l(t)=\left\{\begin{array}{ll}
0 & \text { for } \quad 0 \leq t<12, \\
l_{0} & \text { for } 12 \leq t<24,
\end{array} \quad l(t+24)=l(t) .\right.
$$

Figure 2 shows typical phenomena (i.e., oscillations, mutual synchronization, and locking to light stimulation) of the ODE gene network model with $J=4$ cells, which have slightly different parameter values. In Fig. 2(a), the cells are uncoupled. In this case, the mRNA concentrations $x_{i}(i=1,2,3,4)$ of the four cells oscillate periodically with slightly different periods and amplitudes. Note that this case corresponds to an unrealistic situation since healthy suprachiasmatic nucleus (SCN) neurons are coupled. In Fig. 2(b), the cells are coupled via the mean field $f$ of the neuropeptide $v_{i}$. In this case, the mRNA concentrations $x_{i}$ are synchronized. Note that this case corresponds to the situation where the SCN is put in a dark room. In Fig. 2(c), an external light stimulation $l(t)$ with period 24 hours is applied to the cells. In this case, the periods of oscillations of the mRNA concentrations $x_{i}$ are locked to the period of the light stimulation $l(t)$. Note that this case corresponds to the situation where a sunlight with the period 24 hours is applied to the healthy SCN. Figure 3 shows a bifurcation diagram of the ODE gene network model with respect to the coupling parameter $k$. It has been shown in [7] that, as the coupling parameter $k$ increases, the network exhibits a super-critical Andronov Hopf bifurcation, a fold limit cycle bifurcation, and a sub-critical Andronov Hopf bifurcation as indicated in Fig. 3. In the next section, a special king of cellular automaton is designed and it is shown that the designed automaton can reproduce not only the oscillation, the synchronization, and the locking of the ODE gene network model in Fig. 2 but also the related bifurcation mechanisms in Fig. 3.

\section{Novel asynchronous CA model of gene network}

In this section, a novel asynchronous cellular automaton model of a gene network is proposed, where its vector field is designed based on the ODE gene network model [7]. Figure 4 illustrates a hardware structure of a single cell in the proposed gene network model, where its features are as follows.

Registers storing discrete state variables: The single cell has four registers, which store the following four discrete states variables:

$$
X_{i} \in \boldsymbol{A}_{N}=\{0, \ldots, N-1\}, \quad Y_{i} \in \boldsymbol{A}_{N}, Z_{i} \in \boldsymbol{A}_{N}, V_{i} \in \boldsymbol{A}_{N},
$$

where $X_{i}, Y_{i}, Z_{i}$, and $V_{i}$ correspond to the mRNA concentration $x_{i}$ of the clock gene, the resulting protein concentration $y_{i}$, the inhibitor concentration $z_{i}$, and the neuropeptide concentration $v_{i}$ of the ODE gene network model [7] in Eq. (1), respectively; $N$ is a positive integer determining the resolution of the state space; and $i$ is the cell index.

Registers storing discrete auxiliary variables: The single cell has four registers, that store the following four discrete auxiliary variables $P_{i}, Q_{i}, R_{i}$, and $S_{i}$ of $X_{i}, Y_{i}, Z_{i}$, and $V_{i}$, respectively: 


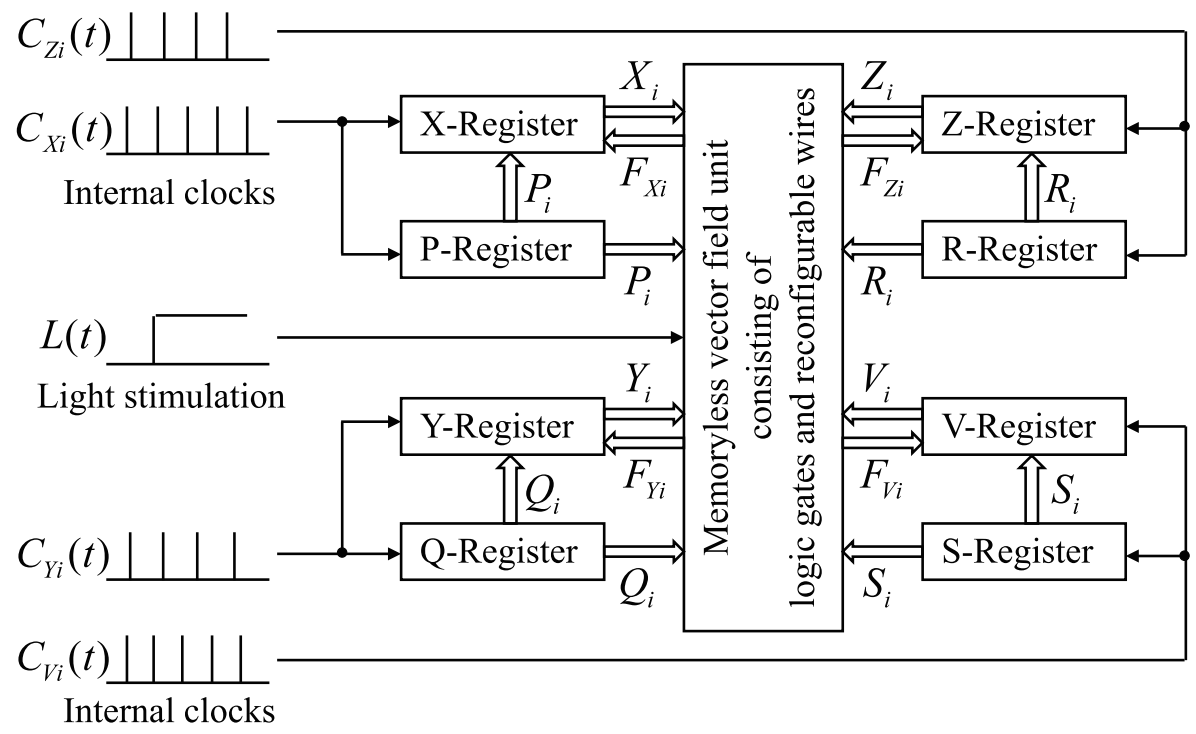

Fig. 4. Hardware structure of a novel asynchronous cellular automaton model of gene network.

$$
P_{i} \in \boldsymbol{A}_{M}=\{0, \ldots, M-1\}, \quad Q_{i} \in \boldsymbol{A}_{M}, \quad R_{i} \in \boldsymbol{A}_{M}, \quad S_{i} \in \boldsymbol{A}_{M},
$$

where $M$ is a positive integers determining the resolution of the state space.

Internal clocks: The single cell has the following four uncoupled asynchronous internal clocks $C_{X_{i}}(t)$, $C_{Y_{i}}(t), C_{Z_{i}}(t)$, and $C_{V_{i}}(t)$ that trigger transitions of the discrete states $\left(X_{i}, P_{i}\right),\left(Y_{i}, Q_{i}\right),\left(Z_{i}, R_{i}\right)$, and $\left(V_{i}, S_{i}\right)$, respectively:

$$
\begin{aligned}
& C_{X_{i}}(t)= \begin{cases}1 & \text { if } t=0, T_{X_{i}}, 2 T_{X_{i}}, \ldots, \\
0 & \text { otherwise, }\end{cases} \\
& C_{Y_{i}}(t)= \begin{cases}1 & \text { if } t=0, T_{Y_{i}}, 2 T_{Y_{i}}, \ldots, \\
0 & \text { otherwise }\end{cases} \\
& C_{Z_{i}}(t)= \begin{cases}1 & \text { if } t=0, T_{Z_{i}}, 2 T_{Z_{i}}, \ldots, \\
0 & \text { otherwise }\end{cases} \\
& C_{V_{i}}(t)= \begin{cases}1 & \text { if } t=0, T_{V_{i}}, 2 T_{V_{i}}, \ldots, \\
0 & \text { otherwise }\end{cases}
\end{aligned}
$$

where $T_{X_{i}}>0, T_{Y_{i}}>0, T_{Z_{i}}>0$, and $T_{V_{i}}>0$ are their periods.

Memoryless vector field unit: The single cell has a memoryless vector field unit consisting of logic gates and reconfigurable wires, which works as the following discrete functions:

$$
\begin{aligned}
& F_{X i}: \boldsymbol{A}_{N} \times \boldsymbol{A}_{N} \times \boldsymbol{A}_{N} \times \boldsymbol{A}_{N} \rightarrow\{-(M-1), \cdots,-1,0,1, \cdots, M-1\}=\boldsymbol{A}_{M}^{ \pm}, \\
& F_{Y i}: \boldsymbol{A}_{N} \times \boldsymbol{A}_{N} \times \boldsymbol{A}_{N} \times \boldsymbol{A}_{N} \rightarrow \boldsymbol{A}_{M}^{ \pm}, \\
& F_{Z i}: \boldsymbol{A}_{N} \times \boldsymbol{A}_{N} \times \boldsymbol{A}_{N} \times \boldsymbol{A}_{N} \rightarrow \boldsymbol{A}_{M}^{ \pm}, \\
& F_{V i}: \boldsymbol{A}_{N} \times \boldsymbol{A}_{N} \times \boldsymbol{A}_{N} \times \boldsymbol{A}_{N} \rightarrow \boldsymbol{A}_{M}^{ \pm},
\end{aligned}
$$

where these functions characterize a nonlinear vector field of the single cell.

External light stimulation: The following external light stimulation $L(t)$ with period 24 hours is applied to the cell.

$$
L(t)=\left\{\begin{array}{ll}
0 & \text { for } \quad 0 \leq t<12, \\
L_{0} \quad \text { for } 12 \leq t<24,
\end{array} \quad L(t+24)=L(t) .\right.
$$

Coupling via mean field of neuropeptide: In the gene network model, the cells are coupled via the following mean field $F$ of the neuropeptide concentrations $V_{i}$ (not illustrated in Fig. 4). 


$$
F=\frac{1}{J} \sum_{i=1}^{J} V_{i}
$$

where $J$ is the number of cells.

In this paper, we propose to design the discrete functions $F_{X_{i}}, F_{Y_{i}}, F_{Z_{i}}$, and $F_{V_{i}}$ in the following manner:

$$
\begin{aligned}
& F_{X_{i}}\left(X_{i}, Y_{i}, Z_{i}, V_{i}\right)= \begin{cases}M-1 & \text { if } \operatorname{Int}\left(\frac{\gamma_{X_{i}}}{g_{x}\left(X_{i}, Y_{i}, Z_{i}, V_{i}\right) T_{X_{i}}}\right) \geq M-1, \\
-(M-1) & \text { if } \operatorname{Int}\left(\frac{\gamma_{X_{i}}}{g_{x}\left(X_{i}, Y_{i}, Z_{i}, V_{i}\right) T_{X_{i}}}\right) \leq-(M-1), \\
\operatorname{Int}\left(\frac{\gamma_{X_{i}}}{g_{x}\left(X_{i}, Y_{i}, Z_{i}, V_{i}\right) T_{X_{i}}}\right) & \text { otherwise. }\end{cases} \\
& F_{Y_{i}}\left(X_{i}, Y_{i}, Z_{i}, V_{i}\right)= \begin{cases}M-1 & \text { if } \operatorname{Int}\left(\frac{\gamma_{Y_{i}}}{g_{y}\left(X_{i}, Y_{i}, Z_{i}, V_{i}\right) T_{Y_{i}}}\right) \geq M-1, \\
-(M-1) & \text { if } \operatorname{Int}\left(\frac{\gamma_{Y_{i}}}{g_{y}\left(X_{i}, Y_{i}, Z_{i}, V_{i}\right) T_{Y_{i}}}\right) \leq-(M-1), \\
\operatorname{Int}\left(\frac{\gamma_{Y_{i}}}{g_{y}\left(X_{i}, Y_{i}, Z_{i}, V_{i}\right) T_{Y_{i}}}\right) \quad \text { otherwise. }\end{cases} \\
& F_{Z_{i}}\left(X_{i}, Y_{i}, Z_{i}, V_{i}\right)= \begin{cases}M-1 & \text { if } \operatorname{Int}\left(\frac{\gamma_{Z_{i}}}{g_{z}\left(X_{i}, Y_{i}, Z_{i}, V_{i}\right) T_{Z_{i}}}\right) \geq M-1, \\
-(M-1) & \text { if } \operatorname{Int}\left(\frac{\gamma_{Z_{i}}}{g_{z}\left(X_{i}, Y_{i}, Z_{i}, V_{i}\right) T_{Z_{i}}}\right) \leq-(M-1), \\
\operatorname{Int}\left(\frac{\gamma_{Z_{i}}}{g_{z}\left(X_{i}, Y_{i}, Z_{i}, V_{i}\right) T_{Z_{i}}}\right) \quad \text { otherwise. }\end{cases} \\
& F_{V_{i}}\left(X_{i}, Y_{i}, Z_{i}, V_{i}\right)= \begin{cases}M-1 & \text { if } \operatorname{Int}\left(\frac{\gamma_{V_{i}}}{g_{v}\left(X_{i}, Y_{i}, Z_{i}, V_{i}\right) T_{V_{i}}}\right) \geq M-1, \\
-(M-1) & \text { if } \operatorname{Int}\left(\frac{\gamma_{V_{i}}}{g_{v}\left(X_{i}, Y_{i}, Z_{i}, V_{i}\right) T_{V_{i}}}\right) \leq-(M-1), \\
\operatorname{Int}\left(\frac{\gamma_{V_{i}}}{g_{v}\left(X_{i}, Y_{i}, Z_{i}, V_{i}\right) T_{V_{i}}}\right) \quad \text { otherwise. }\end{cases} \\
& g_{x}\left(X_{i}, Y_{i}, Z_{i}, V_{i}\right)=\gamma_{X_{i}}\left(A_{1, i} \frac{B_{1, i}^{n}}{B_{1, i}^{n}+Z_{i}^{n}}-A_{2, i} \frac{X_{i}}{B_{2, i}+X_{i}}+A_{c, i} \frac{K F}{B_{c, i}+K F}+L\right) \text {, } \\
& g_{y}\left(X_{i}, Y_{i}, Z_{i}, V_{i}\right)=\gamma_{Y_{i}}\left(B_{3, i} X_{i}-A_{3, i} \frac{Y_{i}}{B_{4, i}+Y_{i}}\right) \text {, } \\
& g_{z}\left(X_{i}, Y_{i}, Z_{i}, V_{i}\right)=\gamma_{Z_{i}}\left(B_{5, i} Y_{i}-A_{4, i} \frac{Z_{i}}{B_{6, i}+Z_{i}}\right) \text {, } \\
& g_{v}\left(X_{i}, Y_{i}, Z_{i}, V_{i}\right)=\gamma_{V_{i}}\left(B_{7, i} X_{i}-A_{5, i} \frac{V_{i}}{B_{8, i}+V_{i}}\right),
\end{aligned}
$$

where Int is the floor function;

$$
\boldsymbol{P}_{i}=\left(A_{1, i}, A_{2, i}, A_{3, i}, A_{4, i}, A_{5, i}, A_{c, i}, B_{1, i}, B_{2, i}, B_{3, i}, B_{4, i}, B_{5, i}, B_{6, i}, B_{7, i}, B_{8, i}, B_{c, i}\right)
$$

are parameters of the $i$-th cell;

$$
\gamma_{X_{i}}, \gamma_{Y_{i}}, \gamma_{Z_{i}}, \gamma_{V_{i}}
$$

are also parameters of the $i$-th cell; and $K$ is a coupling parameter. Let " $t_{+}$" represent " $\lim _{\epsilon \rightarrow 0} t+$ $\epsilon ", \epsilon>0$, and let ":=" represent "instantaneous state transition" hereafter. Then, transitions of 
the discrete state variables $\left(X_{i}, Y_{i}, Z_{i}, V_{i}\right)$ and their discrete auxiliary variables $\left(P_{i}, Q_{i}, R_{i}, S_{i}\right)$ are described by the following equations.

$$
\begin{aligned}
& \text { If } C_{X_{i}}(t)=1 \text { and } P_{i}(t) \geq\left|F_{X_{i}}\right| \text {, then } \\
& X_{i}\left(t_{+}\right):= \begin{cases}X_{i}(t)+1 & \text { if } X_{i}(t) \neq N-1 \text { and } F_{X_{i}} \geq 0, \\
X_{i}(t)-1 & \text { if } X_{i}(t) \neq 0 \text { and } F_{X_{i}}<0, \\
X_{i}(t) & \text { otherwise, }\end{cases} \\
& \text { If } C_{Y_{i}}(t)=1 \text { and } Q_{i}(t) \geq\left|F_{Y_{i}}\right| \text {, then } \\
& Y_{i}\left(t_{+}\right):= \begin{cases}Y_{i}(t)+1 & \text { if } Y_{i}(t) \neq N-1 \text { and } F_{Y_{i}} \geq 0, \\
Y_{i}(t)-1 & \text { if } Y_{i}(t) \neq 0 \text { and } F_{Y_{i}}<0, \\
Y_{i}(t) & \text { otherwise, }\end{cases} \\
& \text { If } C_{Z_{i}}(t)=1 \text { and } R_{i}(t) \geq\left|F_{Z_{i}}\right| \text {, then } \\
& Z_{i}\left(t_{+}\right):= \begin{cases}Z_{i}(t)+1 & \text { if } Z_{i}(t) \neq N-1 \text { and } F_{Z_{i}} \geq 0, \\
Z_{i}(t)-1 & \text { if } Z_{i}(t) \neq 0 \text { and } F_{Z_{i}}<0, \\
Z_{i}(t) & \text { otherwise, }\end{cases} \\
& \text { If } C_{V_{i}}(t)=1 \text { and } S_{i}(t) \geq\left|F_{V_{i}}\right| \text {, then } \\
& V_{i}\left(t_{+}\right):= \begin{cases}V_{i}(t)+1 & \text { if } V_{i}(t) \neq N-1 \text { and } F_{V_{i}} \geq 0, \\
V_{i}(t)-1 & \text { if } V_{i}(t) \neq 0 \text { and } F_{V_{i}}<0, \\
V_{i}(t) & \text { otherwise, }\end{cases} \\
& \text { If } C_{X_{i}}(t)=1 \text {, then } \\
& P_{i}\left(t_{+}\right):= \begin{cases}P_{i}(t)+1 & \text { if } P_{i}(t)<\left|F_{X_{i}}\right|, \\
0 & \text { if } P_{i}(t) \geq\left|F_{X_{i}}\right|,\end{cases} \\
& \text { If } C_{Y_{i}}(t)=1 \text {, then } \\
& Q_{i}\left(t_{+}\right):= \begin{cases}Q_{i}(t)+1 & \text { if } Q_{i}(t)<\left|F_{Y_{i}}\right|, \\
0 & \text { if } Q_{i}(t) \geq\left|F_{Y_{i}}\right|,\end{cases} \\
& \text { If } C_{Z_{i}}(t)=1 \text {, then } \\
& R_{i}\left(t_{+}\right):= \begin{cases}R_{i}(t)+1 & \text { if } R_{i}(t)<\left|F_{Z_{i}}\right|, \\
0 & \text { if } R_{i}(t) \geq\left|F_{Z_{i}}\right|,\end{cases} \\
& \text { If } C_{V_{i}}(t)=1 \text {, then } \\
& S_{i}\left(t_{+}\right):= \begin{cases}S_{i}(t)+1 & \text { if } S_{i}(t)<\left|F_{V_{i}}\right|, \\
0 & \text { if } S_{i}(t) \geq\left|F_{V_{i}}\right| .\end{cases}
\end{aligned}
$$

Figure 5 shows typical asynchronous transitions of the discrete state variables $\left(X_{i}, Y_{i}, Z_{i}, V_{i}\right)$ and their discrete auxiliary variables $\left(P_{i}, Q_{i}, R_{i}, S_{i}\right)$. Figure 6 shows typical phenomena (i.e., oscillations, mutual synchronization, and locking to light stimulation) of the proposed gene network model with $J=4$ cells which have slightly different parameter values. In Fig. 6(a), the cells are uncoupled. In this case, the mRNA concentrations $X_{i}(i=1,2,3,4)$ of the four cells oscillate periodically with slightly different periods and amplitudes. In Fig. 6(b), the cells are coupled via the mean field $F$ of the neuropeptide $V_{i}$. In this case, the mRNA concentrations $X_{i}$ are synchronized. In Fig. 6(c), the external light stimulation $L(t)$ with period 24 hours is applied to the cells. In this case, the periods of oscillations of the mRNA concentrations $X_{i}$ are locked to the period of the light stimulation $L(t)$. Comparing Fig. 6 with Fig. 2, it can be confirmed that the proposed gene network model can reproduce the typical phenomena of the ODE gene network model. Figure 7 shows a bifurcation diagram of the proposed gene network model with respect to the coupling parameter $K$. It can bee 


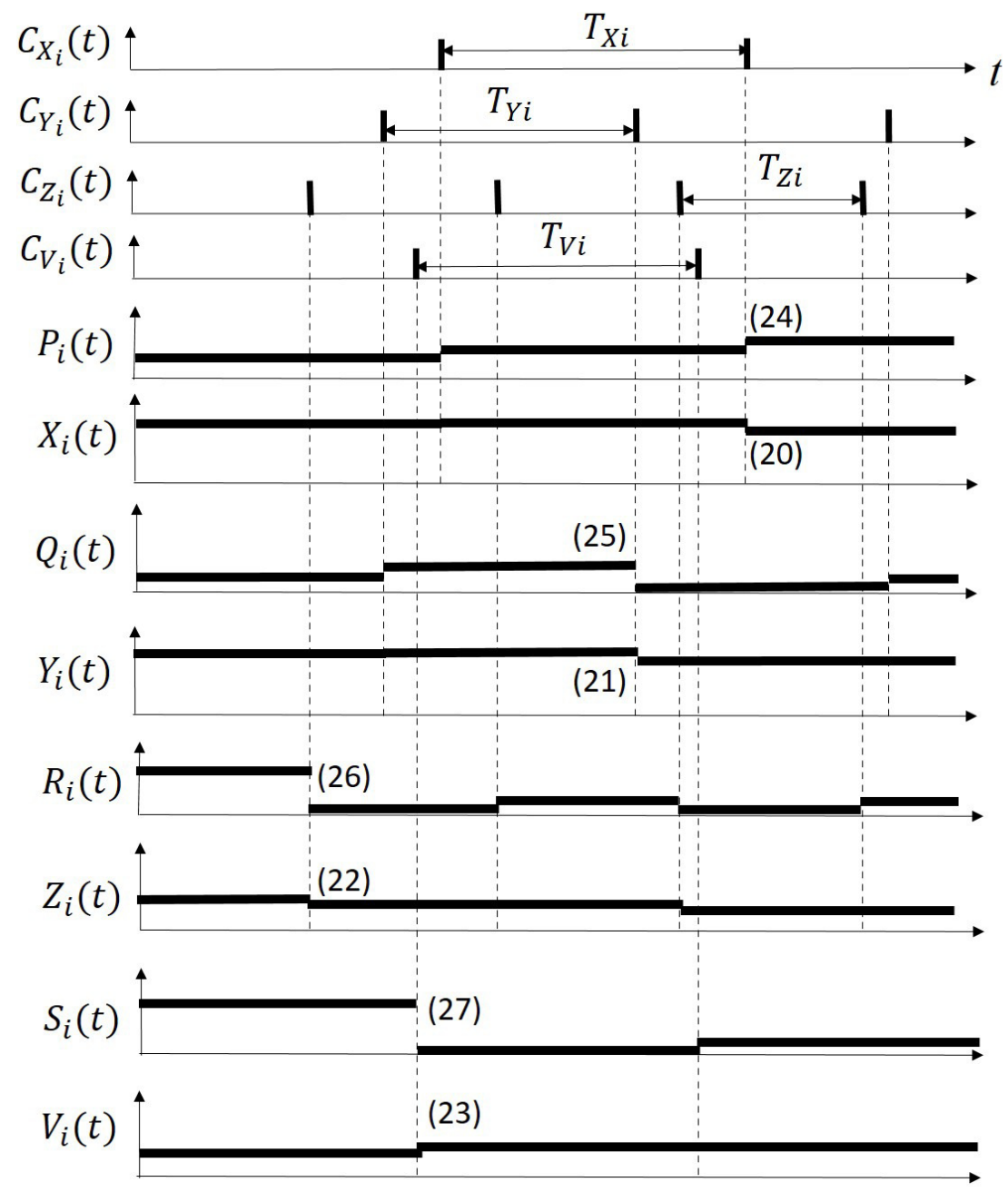

Fig. 5. Typical asynchronous transitions of the discrete state variables $\left(X_{i}, Y_{i}, Z_{i}, V_{i}\right)$ and their discrete auxiliary variables $\left(P_{i}, Q_{i}, R_{i}, S_{i}\right)$. The labels (20)-(27) indicate the asynchronous transitions described in Eqs. (20)-(27), respectively.

seen that, as the coupling parameter $K$ increases, the proposed model exhibits bifurcations similar to the super-critical Andronov Hopf bifurcation, the fold limit cycle bifurcation, and the sub-critical Andronov Hopf bifurcation of the ODE gene network model in Fig. 3. By using extensive numerical experiments, we have confirmed that occurrence mechanisms of the above bifurcations are qualitatively similar (e.g., birth of a separatrix via the sub-critical Andronov Hopf bifurcation and its vanish via the fold limit cycle bifurcation; and non-birth of such a separatrix via the super-critical Andronov Hopf bifurcation) to those of the ODE gene network model. These results give an important implication: although the proposed model is a special kind of cellular automaton having the discrete state space $\boldsymbol{A}_{N} \times \boldsymbol{A}_{N} \times \boldsymbol{A}_{N} \times \boldsymbol{A}_{N} \times \boldsymbol{A}_{M} \times \boldsymbol{A}_{M} \times \boldsymbol{A}_{M} \times \boldsymbol{A}_{M}$, it may be able to reproduce bifurcation mechanisms of a nonlinear ODE such as existence of a 2-dimensional center manifold in a high-dimensional discrete state space, non-hyperbolicity and non-degeneracy of an equilibrium state on the center manifold, and transversality with respect to change of parameter value. More detailed bifurcation analyses in the asynchronous cellular automaton will be presented in a future paper that concentrates on analyses.

\section{Remark}

Let us give intuitive comparisons of the proposed gene network model and a numerical integration of the ODE gene network model.

- The numerical integration such as the Euler formula typically uses multipliers, which consume a lot of transistors. In the numerical integration, the time axis is discretized, typically with equal 


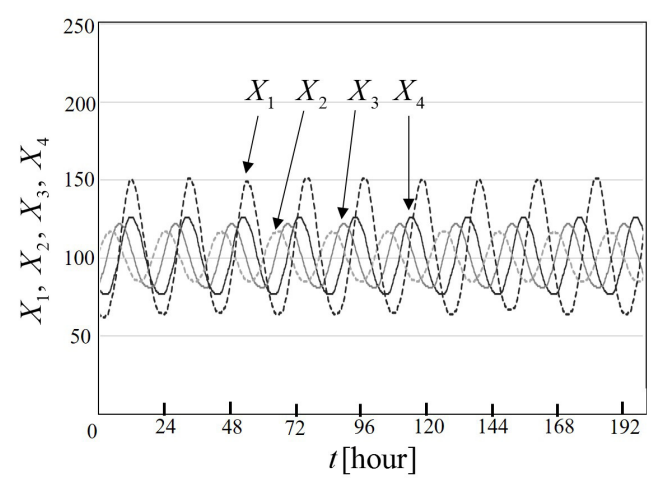

(a) Uncoupled four cells.

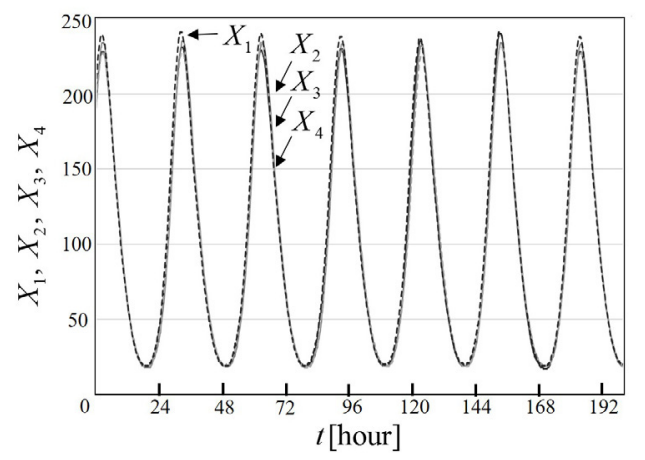

(b) Four cells coupled via mean field $F$ of neuropeptide $V_{i}$.

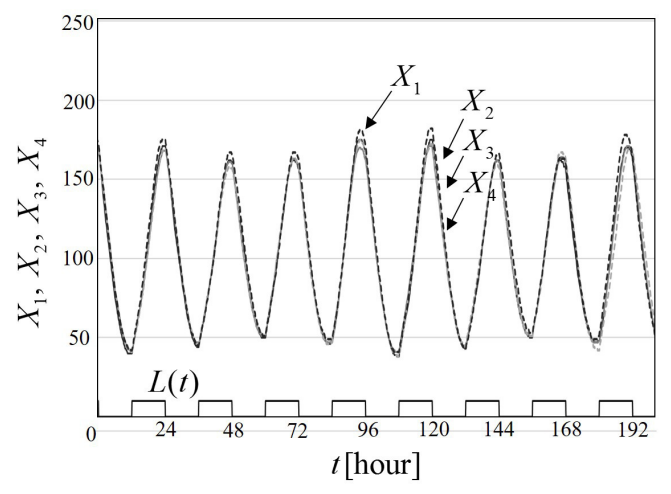

(c) Coupled four cells under light stimulation $L(t)$ with period 24 hours.

Fig. 6. Typical phenomena of the proposed gene network model with $J=4$ cells. The four cells have slightly different parameter values: the parameter values $\boldsymbol{P}_{1}, \boldsymbol{P}_{2}, \boldsymbol{P}_{3}$, and $\boldsymbol{P}_{4}$ are identical with the parameter values $\boldsymbol{p}_{1}, \boldsymbol{p}_{2}, \boldsymbol{p}_{3}$, and $\boldsymbol{p}_{4}$ of the ODE gene network model in Fig. 2, respectively. ( $\left.N, M, \gamma_{X_{i}}, \gamma_{Y_{i}}, \gamma_{X_{i}}, \gamma_{V_{i}}\right)=(2048,2048,800,700,300,800)$. $\left(T_{X_{i}}, T_{Y_{i}}, T_{Z_{i}}, T_{V_{i}}\right)=(0.0097,0.0053,0.0007,0.0089)$. (a) Oscillations. The cells are uncoupled. $K=0, L_{0}=0$. (b) Mutual synchronization. The cells are coupled via the mean field $F$ of the neuropeptide $V_{i} . K=0.5, L_{0}=0$. (c) Locking of period to stimulation period. The cells are coupled via the mean field $F$ of the neuropeptide $V_{i}$ and an external light stimulation $L$ with period 24 hours is applied to the cells. $K=0.5, L_{0}=0.01$.

width called a step size. Hence, intuitively speaking, utility rate of hardware of the numerical integration is high (meaning a lot of transistors) and utility rate of the time axis is low (meaning very restricted discretized points on the time axis).

- The proposed model consumes fewer transistors since resolutions of the discrete functions $F_{X i}$, 


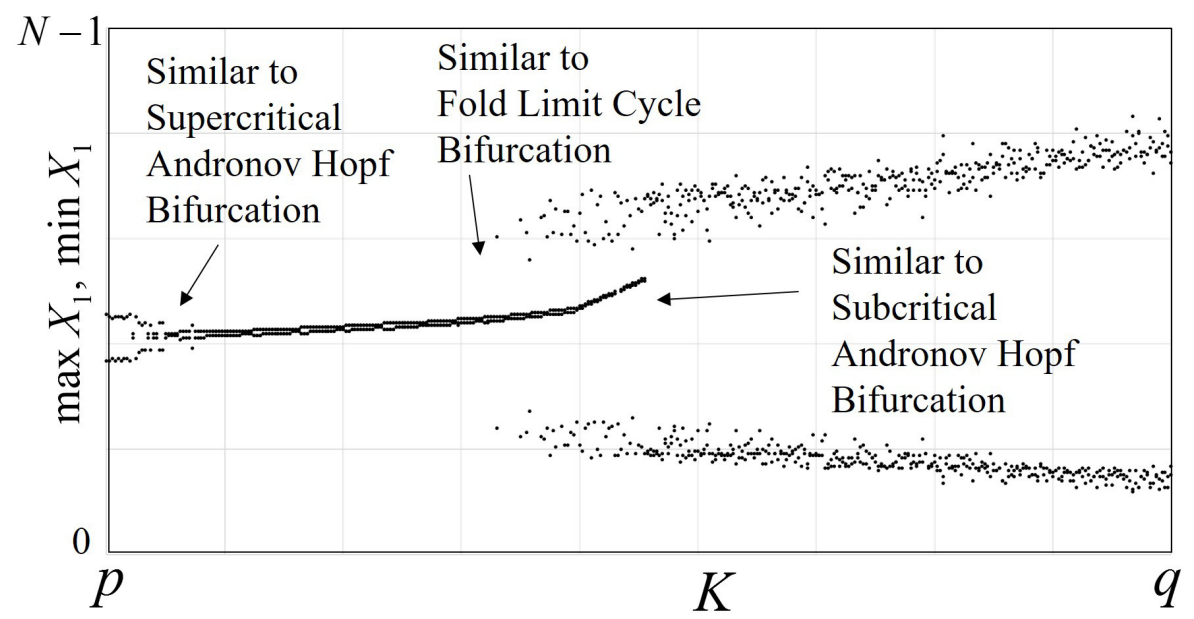

Fig. 7. One parameter bifurcation diagram of the proposed gene network model. $J=2 . K$ is the rescaled coupling parameter, where $p=0.05$ and $q=0.5 . p_{1}=(0.707,0.3535,0.3535,0.3535,1.01,0.4,1,1,0.707,1,0.707$, $1,0.3535,1,1) . \quad p_{2}=(0.7,0.35,0.35,0.35,1,0.4,1,1,0.7,1,0.7,1$, $0.35,1,1) . \quad\left(N, M, \gamma_{X_{i}}, \gamma_{Y_{i}}, \gamma_{X_{i}}, \gamma_{V_{i}}\right)=(2048,2048,800,700,300,2200)$. $\left(T_{X_{1}}, T_{Y_{1}}, T_{Z_{1}}, T_{V_{1}}\right)=(0.0101,0.0031,0.0011,0.0089) .\left(T_{X_{2}}, T_{Y_{2}}, T_{Z_{2}}, T_{V_{2}}\right)=$ (0.0091, 0.0021, 0.0011, 0.0089).

$F_{Y i}, F_{Z i}$, and $F_{V i}$ (which determine the vector field) are much lower than those of corresponding functions of the numerical integration. Actually, the discrete functions of the proposed model are implemented by look-up tables and adders without a multiplier. In the proposed model, the discrete states can transit at various points on the time axis as illustrated in Fig. 5. Hence, intuitively speaking, utility rate of hardware of the proposed model is low (meaning fewer transistors) and utility rate of the time axis is high (meaning various points on the time axis).

- As a result, intuitively speaking, the asynchronous cellular automaton gene network model is expected to have better performance in terms of precision of reproduction of behaviors of the ODE model than a synchronous cellular automaton gene network model and the numerical integration gene network model. This expectation is validated in the next section.

\section{FPGA implementations and comparison}

Figure 8 shows numerical simulation results of the ODE gene network model [7] in Eq. (1), which are obtained by using floating point numbers with the double precision. These phenomena are used as references for comparison of an FPGA-implemented proposed gene network model and an FPGAimplemented ODE gene network model, i.e., the FPGA-implemented models are supposed to try to reproduce the simulation results in Fig. 8.

Recall that the state transitions of the proposed gene network model are described by Eqs. (20)(27). These equations are rewritten as a VHDL code, which is compiled into a bitstream file, and the resulting file is downloaded to a field programmable gate array (ab. FPGA). The memoryless vector field unit (i.e., the discrete functions $F_{X i}, F_{Y i}, F_{Z i}$, and $F_{V i}$ ) is implemented by combinations of look-up tables, adders, and subtractors, where the nonlinear terms in the discrete functions are implemented by the look-up tables. Hence no DSP slice (which is a multiplier consisting of a lot of transistors) of the FPGA is used. Since the FPGA device and the HDL compiler used in this paper (see Table I) do not support asynchronous triggering, the internal clocks $C_{X_{i}}(t), C_{Y_{i}}(t), C_{Z_{i}}(t)$, and $C_{V_{i}}(t)$ are generated from a common clock with high frequency (100[MHz]). Since the frequency of the common clock is much higher than the frequencies of the internal clocks, the resulting behavior of the implemented circuit can be regarded to be almost identical with the asynchronous behavior of the proposed gene network model, and we have confirmed by intensive experiments that they are actually almost identical. Figure 9 shows typical phenomena of the FPGA-implemented proposed gene network model, where the parameter values $\boldsymbol{P}_{1}, \boldsymbol{P}_{2}, \boldsymbol{P}_{3}$, and $\boldsymbol{P}_{4}$ are identical with the parameter 

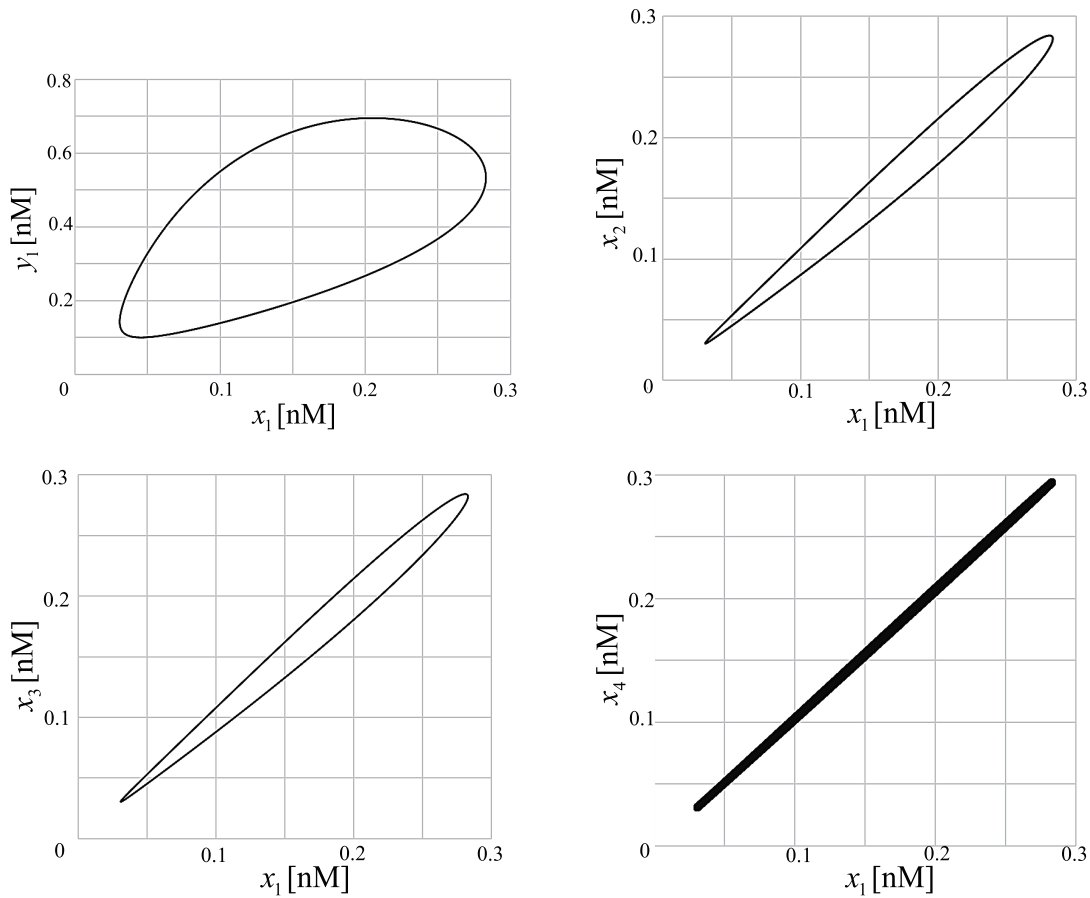

(a) Four cells coupled via the mean field $f$.
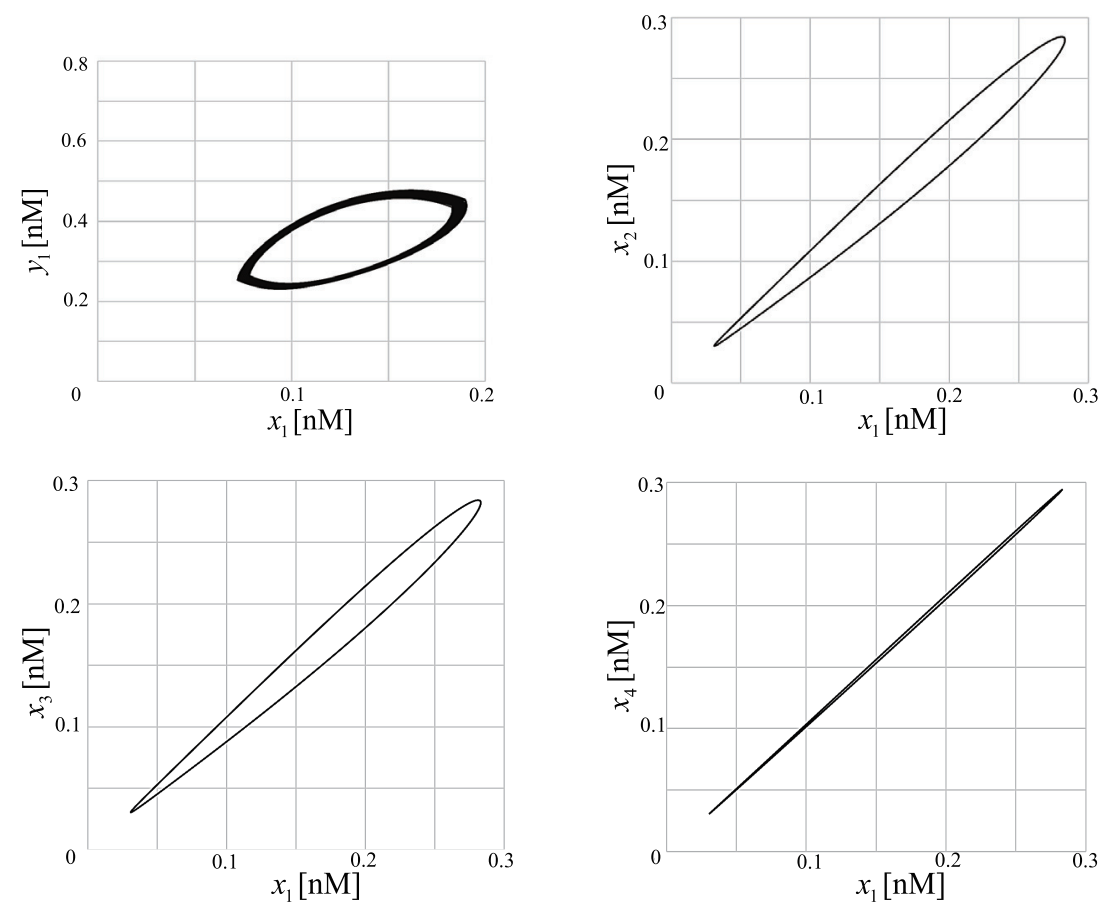

(b) Four cells coupled via the mean field $f$ and the light stimulation $l(t)$ is applied.

Fig. 8. References for comparison: numerical simulation results of the ODE gene network model [7] in Eq. (1) by using floating point numbers with the double precision. Phase planes of the ODE gene network model with $J=4$ cells. The parameter values are identical with those in Fig. 2. (a) The cells are coupled via the mean field $f$. (b) The cells are coupled via the mean field $f$ and the light stimulation $l(t)$ is applied.

values $\boldsymbol{p}_{1}, \boldsymbol{p}_{2}, \boldsymbol{p}_{3}$, and $\boldsymbol{p}_{4}$ of the ODE gene network model in Fig. 2, respectively. Also, the parameter values $\left(N, M, T_{X_{i}}, T_{Y_{i}}, T_{Z_{i}}, T_{V_{i}}, \gamma_{X_{i}}, \gamma_{Y_{i}}, \gamma_{X_{i}}, \gamma_{V_{i}}\right)$ of the proposed gene network model are identical with those in Fig. 6. Comparing Fig. 9 with Fig. 8, it can be confirmed that the FPGA- 
Table I. Comparison of implementation costs. Xilinx's HDL Compiler Vivado 2015.04 (64-bit) and Xilinx's FPGA XC7A100T-1CSG324C are used for implementation.

\begin{tabular}{|c|c|c|c|c|c|}
\hline & $\begin{array}{l}\text { Resolution of } \\
\text { discrete state }\end{array}$ & $\begin{array}{l}\text { Number of } \\
\text { flip-flops in } \\
\text { FPGA slices }\end{array}$ & $\begin{array}{l}\text { Number of } \\
\text { look-up tables } \\
\text { in FPGA slices }\end{array}$ & $\begin{array}{l}\text { Number of } \\
\text { FPGA slices }\end{array}$ & $\begin{array}{l}\text { Number of } \\
\text { DSP slices }\end{array}$ \\
\hline Proposed model & $N=M=2048$ & 2,027 & 18,260 & 5,301 & 0 \\
\hline ODE model & 27bit fixed point & 604 & 53,571 & 14,628 & 0 \\
\hline ODE model & 27bit fixed point & 482 & 44,700 & 12,244 & 40 \\
\hline
\end{tabular}

implemented proposed gene network model can reproduce the typical phenomena of the ODE gene network model. Note that the resolution $(M, N)$ of the discrete state space of the proposed model is decreased as small as possible under the condition that the FPGA-implemented proposed model qualitatively reproduces the phenomena in Fig. 8 (i.e., the proposed model exhibits oscillations with slightly different periods, mutual synchronization, and locking to the light stimulation, where each waveform is similar to a rescaled version of corresponding waveform in Fig. 8), where the resulting resolution $(M, N)$ is shown in Table I. Also, Table I summarizes implementation cost (i.e., the number of flip-flops, look-up tables, and FPGA slices) of the proposed model. Note that the eight discrete states $\left(X_{i}, Y_{i}, Z_{i}, V_{i}, P_{i}, Q_{i}, R_{i}, S_{i}\right)$ of the four neurons are implemented by $11 \times 8 \times 4=352$ flip-flops in the FPGA slices and the other 1,675 flip-flops are used as input and output buffers of the memoryless vector field unit.

For comparison, the ODE gene network model is also implemented on the same FPGA and by the same HDL compiler. A numerical integration formula (forward Euler formula) of Eq. (1) is rewritten as a VHDL code by using fixed point numbers. Figure 10 shows typical time waveforms of the FPGAimplemented ODE gene network model, where the tilde " " implies "implemented by a fixed point number" and the parameter values $\boldsymbol{p}_{1}, \boldsymbol{p}_{2}, \boldsymbol{p}_{3}$, and $\boldsymbol{p}_{4}$ are identical with those in Fig. 2 . Note that the bit-length of the fixed point numbers is decreased as short as possible under the condition that the FPGA-implemented ODE gene network model qualitatively reproduces the phenomena in Fig. 8, where the resulting bit-length is shown in Table I. As shown in Table I, the ODE gene network model is implemented in two ways: using DSP slices and using no DSP slice. It can be seen that the proposed gene network model consumes much less hardware resource (i.e., much fewer number of FPGA slices and DSP slices) compared to the ODE gene network model. Note that the number of look-up tables affects the number of FPGA slices more strongly than the number of flip-flops. So, the fact that the proposed model consumes fewer look-up tables is more significant than the fact that the proposed model consumes more flip-flops.

Further comparisons on advantage of the asynchronous state transitions are given in Fig. 11. Figure 11(a) shows a simulation result of the ODE gene network model [7] in Eq. (1) by using floating point numbers with the double precision and now it is used as a reference phenomenon to be reproduced by the following three FPGA-implemented gene network models. Figure 11(b) shows a phase plane trajectory of an FPGA-implemented proposed asynchronous cellular automaton gene network model. It can be seen that the proposed model can reproduce the numerical simulation result in Fig. 11(a). Figure 11(c) shows a phase plane trajectory of an FPGA-implemented synchronous cellular automaton gene network model, where its hardware structure is the same as that of (b) but the transitions of the all discrete states are triggered by a single clock. It can be seen that the amplitude of the oscillation is too small compared to that in Fig. 11(a) and thus it can be said that precision of reproduction of the behavior of the ODE gene network model is low. Figure 11(d) shows a phase plane trajectory of an FPGA-implemented forward Euler formula of the ODE gene network model by using fixed point numbers. It can be seen that the forward Euler formula cannot reproduce the numerical simulation result in Fig. 11(a). As a result, it can be concluded that the proposed asynchronous cellular automaton model has better performance in terms of precision of reproduction of the behaviors of the ODE model and thus the intuitive expectation in the Remark is now validated. 

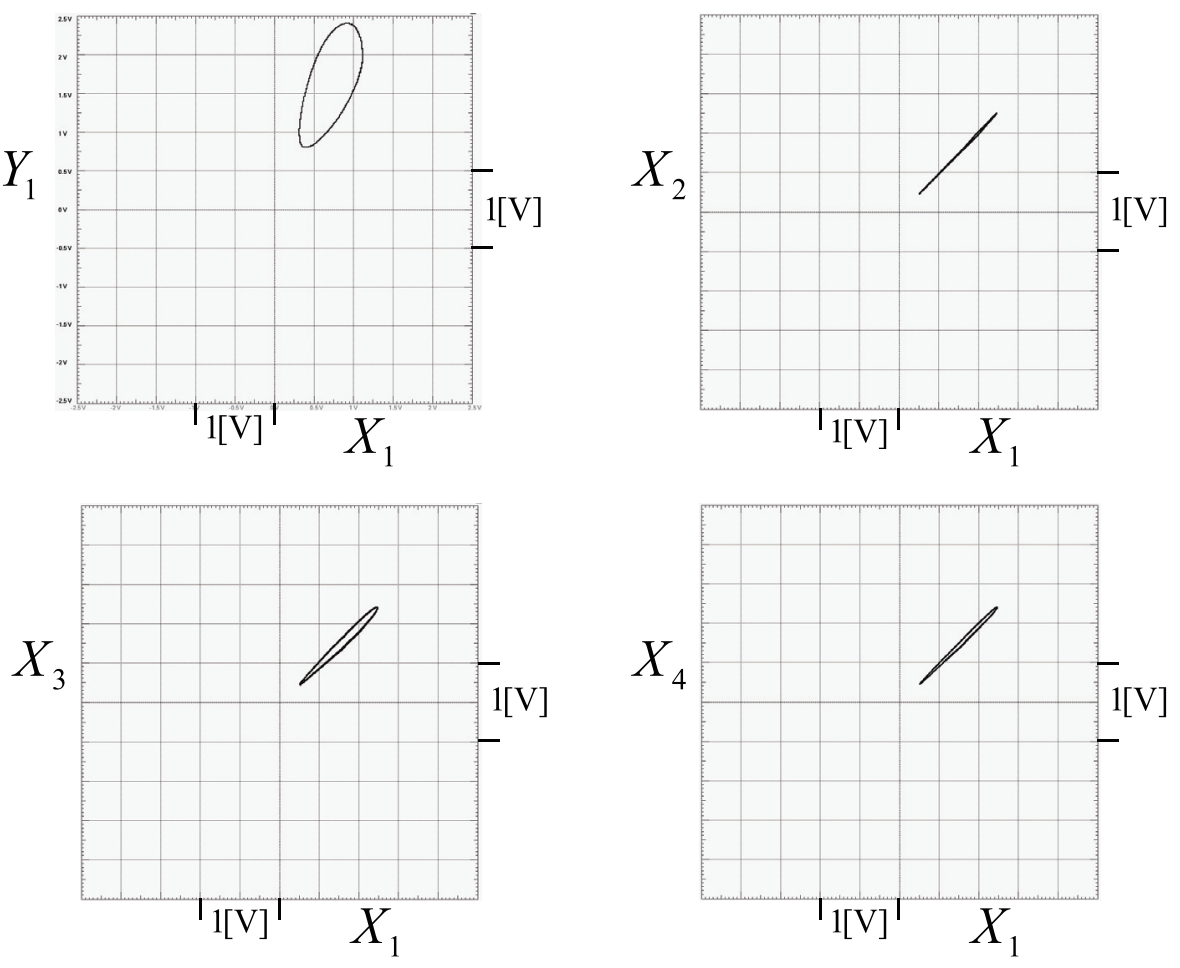

(a) Four cells coupled via the mean field $F$.


(b) Four cells coupled via the mean field $F$ and the light stimulation $L(t)$ is applied.

Fig. 9. Experimental measurements of the FPGA-implemented proposed gene network model. The parameter values $\boldsymbol{P}_{1}, \boldsymbol{P}_{2}, \boldsymbol{P}_{3}$, and $\boldsymbol{P}_{4}$ are identical with the parameter values $\boldsymbol{p}_{1}, \boldsymbol{p}_{2}, \boldsymbol{p}_{3}$, and $\boldsymbol{p}_{4}$ of the ODE gene network model in Fig. 2, respectively. The parameter values $\left(N, M, \gamma_{X_{i}}, \gamma_{Y_{i}}, \gamma_{X_{i}}, \gamma_{V_{i}}\right)$ $=(2048,2048,800,700,300,800)$ and $\left(T_{X_{i}}, T_{Y_{i}}, T_{Z_{i}}, T_{V_{i}}\right)=(0.0097,0.0053$, $0.0007,0.0089)$ are identical with those in Fig. 6 . The hardware cost are summarized in Table I. (a) The cells are coupled via the mean field $F$. (b) The cells are coupled via the mean field $F$ and the light stimulation $L(t)$ are applied. 

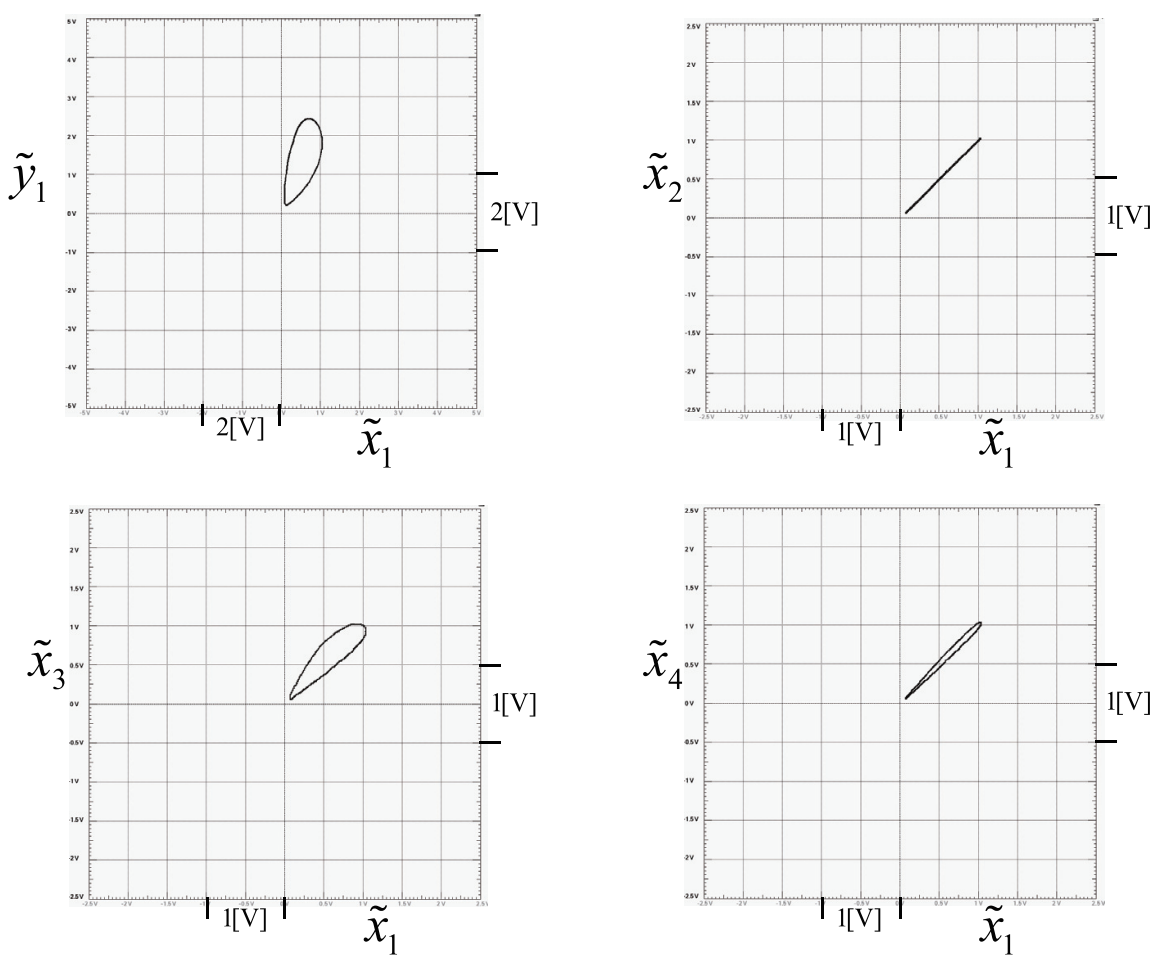

(a) Four cells coupled via the mean field $\tilde{f}$.
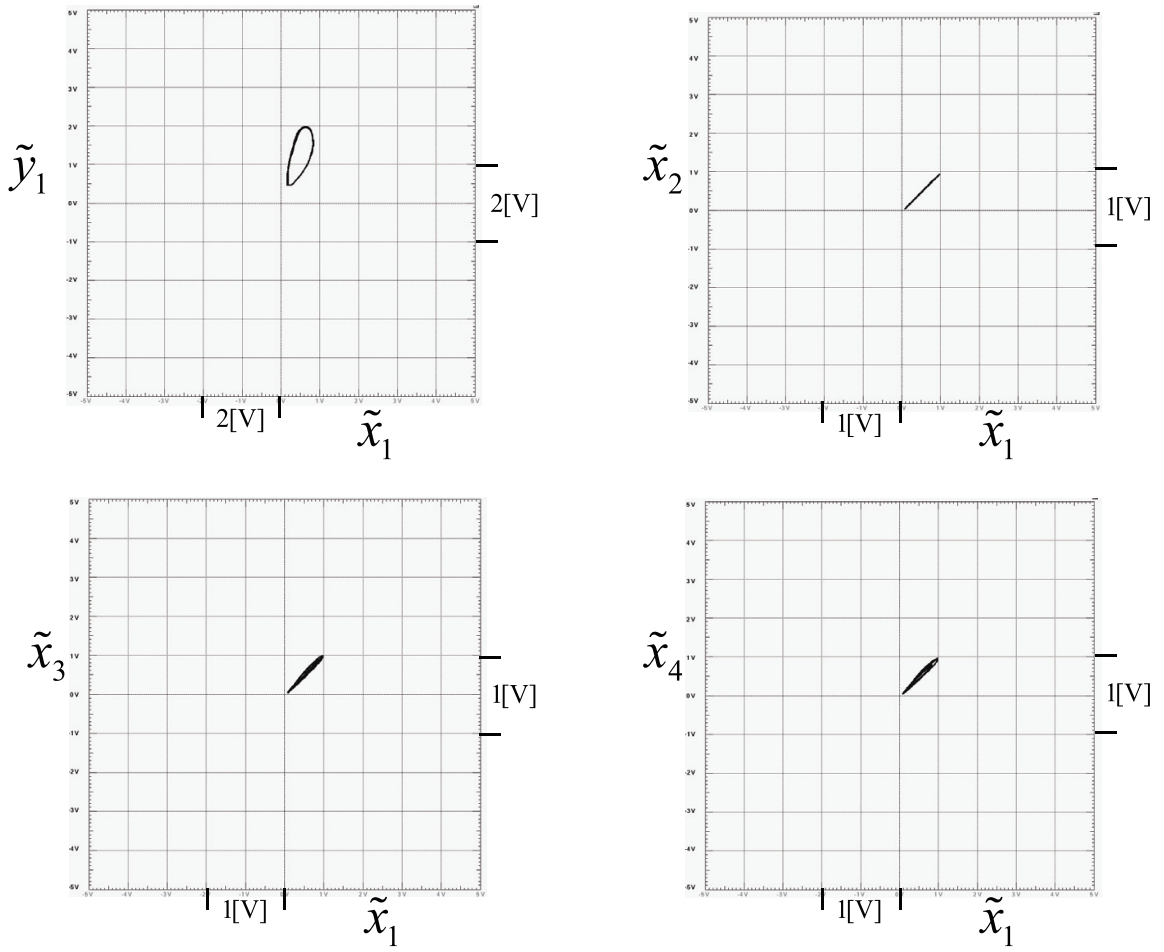

(b) Four cells coupled via the mean field $\tilde{f}$ and the light stimulation $\tilde{l}(t)$ is applied.

Fig. 10. Experimental measurements of the FPGA-implemented ODE gene network model in Eq. (1). The parameter values are identical with those in Fig. 2, where the last digits of the parameter values are rounded up. The tilde " " implies "implemented by a fixed point number." Two cases where DSP slices are used and not used are implemented, where the hardware costs for these cases are summarized in Table I. (a) The cells are coupled via the mean field $\tilde{f}$. (b) The cells are coupled via the mean field $\tilde{f}$ and the light stimulation $\tilde{l}(t)$ is applied. 


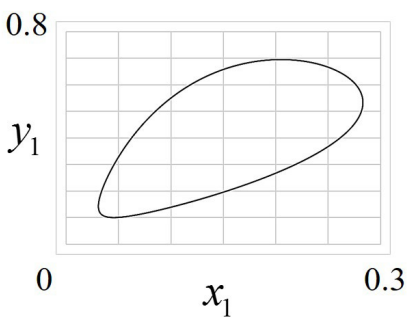

(a) Euler formula by floating point with double precision.

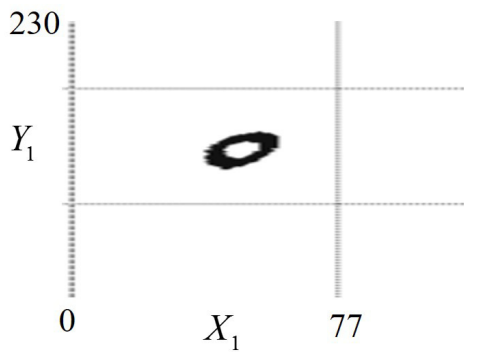

(c) Failure of reproduction of (a) by synchronous cellular automaton model.

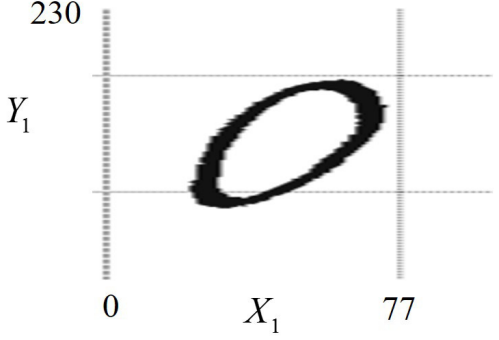

(b) Reproduction of (a) by proposed asynchronous cellular automaton model.

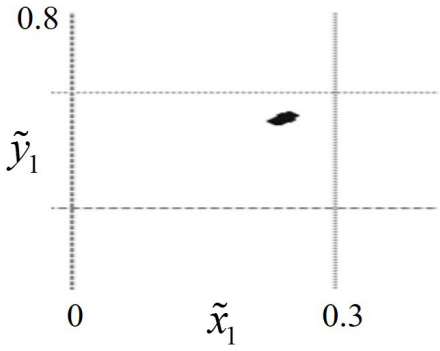

(d) Failure of reproduction of (a) by Euler formula with fixed point.

\begin{tabular}{llll}
\hline & $\begin{array}{l}\text { (b) Asynchronous } \\
\text { cellular automaton }\end{array}$ & $\begin{array}{l}\text { (c) Synchronous } \\
\text { cellular automaton }\end{array}$ & (d) Euler formula \\
\hline $\begin{array}{l}\text { Number of } \\
\text { FPGA slices }\end{array}$ & 5,301 & 5,234 & 7,360 \\
Precision of & (see note 1) & (see note 2) \\
Reproduction of (a) & & Low & Very low \\
\hline
\end{tabular}

Fig. 11. An example of advantage of the asynchronous state transition. (a) Reference for comparison: numerical simulation result of a forward Euler formula of the ODE gene network model [7] in Eq. (1) by using floating point numbers with double precision. $J=1$. The parameter values are identical with those in Fig. 2. (b) FPGA-implemented asynchronous cellular automaton gene network model. $J=1$. The parameter values are identical with those in Fig. 6 . The periods of the internal clocks are $\left(T_{X_{i}}, T_{Y_{i}}, T_{Z_{i}}, T_{V_{i}}\right)=(0.0097,0.0053$, 0.0007, 0.0089). (c) FPGA-implemented synchronous cellular automaton gene network model. $J=1$. Note 1: the structure of the hardware is the same as (b) and the synthesized circuit consumes almost identical number of slices. The internal clocks have the same period $T_{X_{i}}=T_{Y_{i}}=T_{Z_{i}}=T_{V_{i}}=0.001$. The other parameter values are identical with those in (b). (d) FPGA-implemented forward Euler formula of the ODE gene network model by using fixed point numbers. $J=1$. The parameter values are identical with those in (a). Note 2 : the bit length of the fixed point numbers is 18 , which is the possible minimum value so that the circuit can be synthesized by the HDL compiler.

\section{Conclusions}

In this paper, the gene network model based on the asynchronous cellular automaton dynamics is proposed. It was shown that the proposed gene network model can reproduce the typical phenomena of the ODE gene network model such as the oscillations, the mutual synchronization, and the locking to light stimulation. It was also shown that the proposed gene network model can be implemented on the FPGA by using much less hardware resource compared to the ODE gene network model. Hence the results of the paper may contribute to develop other gene network models that consume less hardware resource compared to ordinary differential equation gene network models. Future problems 
include: (a) analysis of power efficiency of the proposed model, (b) detailed bifurcation analysis of small scale gene networks, (c) analysis of large scale gene networks, (d) development of a hardwarebased large scale gene network simulator. This work was partially supported by JSPS KAKENHI Grant Number 15K00352. The authors would like to thank Mr. Ryota Araki for providing preliminary experimental data on this work.

\section{References}

[1] M.E. Ahsen, H. Ozbay, and S.-I. Niculescu, "Analysis of deterministic cyclic gene regulatory network models with delays," Birkhauser Basel (Springer Briefs in Control, Automation and Robotics), 2015.

[2] E. Barillot, et. al., "Computational systems biology of cancer," CRC Press, 2012.

[3] B. Ingalls, "Mathematical modeling in systems biology: An introduction," The MIT Press, 2013.

[4] J. DiStefano III, "Dynamic systems biology modeling and simulation," Academic Press, 2015.

[5] E.R. Kandel, et. al., "Principles of neural science," McGraw-Hill, 2003.

[6] P.D. Smolen and J.H. Byrne, "Circadian rhythm models," Encyclopedia of Neuroscience, pp. 957-963, 2009.

[7] D. Gonze, et. al., "Spontaneous synchronization of coupled circadian oscillators," Biophysical Journal, vol. 89, no. 1, pp. 120-129, 2005.

[8] K. Isobe and H. Torikai, "A novel hardware-efficient asynchronous cellular automaton model of spike-timing dependent synaptic plasticity," IEEE Trans. CAS-II, vol. 63, no. 6, pp. 603-607, 2016.

[9] T. Matsubara, H. Torikai, and T. Hishiki, "A generalized rotate-and-fire digital spiking neuron model and its on-FPGA learning," IEEE Trans. CAS-II, vol. 58, no. 10, pp. 677-681, 2011.

[10] T. Hishiki and H. Torikai, "A novel rotate-and-fire digital spiking neuron and its neuron-like bifurcations and responses," IEEE Trans. NN, vol. 22, no. 5, pp. 752-767, 2011.

[11] S. Hashimoto and H. Torikai, "A novel hybrid spiking neuron: Bifurcations, responses, and on-chip learning," IEEE Trans. CAS-I, vol. 57, no. 8, pp. 2168-2181, 2010.

[12] T. Matsubara and H. Torikai, "Asynchronous cellular automaton based neuron: Theoretical analysis and on-FPGA learning," IEEE Trans. NNLS, vol. 24, no. 5, pp. 736-748, 2013.

[13] T. Matsubara and H. Torikai, "An asynchronous recurrent network of cellular automaton-based neurons and its reproduction of spiking neural network activities," IEEE Trans. NNLS, vol. 27, no. 4, pp. 836-852, 2016.

[14] N. Shimada and H. Torikai, "A novel asynchronous cellular automaton multi-compartment neuron model," IEEE Trans. CAS-II, vol. 62, no. 8, pp. 776-780, 2015.

[15] T. Noguchi and H. Torikai "Ghost stochastic resonance from asynchronous cellular automaton neuron model," IEEE Trans. CAS-II, vol. 60, no. 2, pp. 111-115, 2013.

[16] T. Yoshimoto and H. Torikai, "A hardware-efficient gene network model based on asynchronous bifurcation processor," Proc. NOLTA, 2016.

[17] R. Araki, H. Torikai, and T. Yoshimoto, "A novel gene network model based on nonlinear dynamics of asynchronous cellular automaton," Proc. IJCNN (submitted). 